\title{
La part du mâle dans les avortements spontanés a répétition
}

\author{
M. Auroux \\ Biologie de la Reproduction et du Développement, CHU BICETRE \\ (Université Paris-Sud) 94275 Kremlin Bicêtre.
}

\section{RESUME}

Les facteurs paternels, particulièrement génétiques, jouent un rôle dans les avortements spontanés à répétition (ASR). Le vieillissement paternel, dont on sait qu'il accroît le risque d'apparition de syndromes autosomiques dominants chez le conceptus, pourrait augmenter le risque d'ASR. Toutefois cette éventualité n'est pas clairement établie. Dans une perspective générale, le rôle d'un très jeune âge paternel devrait aussi être envisagé

Mots-clés : âge paternel, avortements spontanés à répétition, anomalies caryotypiques, mutations.

L'âge paternel joue-t-il un rôle dans les avortements spontanés à répétition (ASR) ? Compte tenu des modifications socio-économiques des pays industrialisés, qui voient l'élévation progressive de l'âge des couples à la naissance du premier enfant et l'augmentation de fréquence des unions entre hommes "mûrs" et femmes plus jeunes, il est évident que c'est une question que l'on peut se poser. Et cela d'autant plus que l'on sait que le vieillissement paternel peut retentir sur la qualité du conceptus. Mais il faut d'abord considérer, indépendamment de son âge, le rôle de l'homme dans les ASR.

\section{LE RÔLE DU MÂLE DANS LES ASR.}

On a dit que, dans l'espèce humaine, $70 \%$ des oeufs mouraient avant la fin du premier trimestre [1]. Les aneuploïdies seraient les grandes responsables de cette hécatombe avec au premier chef les trisomies (50 à 60 $\%$ ), suivies des polyploïdies ( 20 à $25 \%$ ) et des monosomies X (15 à $25 \%$ ) [2]. Dans ce contexte, comme le font remarquer certains auteurs [3], le rôle du père dans les avortements est manifeste puisque l'absence de l'X dans la plupart des abortus X0 est d'origine paternelle. L'argument de la dispermie aboutissant à l'avortement d'un oeuf triploïde est plus discutable car la pénétration de deux spermatozoïdes normaux peut être due à une insuffisance ovocytaire. Quoi qu'il en soit, si ces anomalies peuvent expliquer des avortements accidentels, elles n'expliquent pas les ASR. Quelques enquêtes ou résultats expérimentaux sont toutefois parvenus à mettre assez clairement le mâle en cause dans ce type d'avortement. C'est ainsi que, dans une population de couples présentant des ASR on a noté, dans les spermatozoïdes des hommes de 28 à 45 ans concernés, une augmentation de fréquence des cassures chromosomiques [4] et de l'hétérogénéité nucléaire [5] (nous ne parlerons ici ni des problèmes immunologiques, ni des anomalies du spermogramme ou du détail des anomalies génétiques, qui font l'objet d'articles séparés). On sait par ailleurs qu'il existe des 
professions exposées. Les hommes en contact avec le mercure, les solvants, les gaz anesthésiques et les pesticides forment des couples où les avortements sont particulièrement fréquents $[6,7]$. Ce sont les pesticides utilisés dans les bananeraies qui, après qu'on ait constaté une chute anormale de la natalité, ont été à l'origine de l'alerte. Chez l'animal, une expérience assez démonstrative permet de mettre des mutations en cause [8] : administrée au rat mâle la procarbazine, antimitotique dont on connait le pouvoir mutagène, entraîne une augmentation significative des pertes embryonnaires après l'implantation, l'anomalie concernant aussi la F2 issue des ratons mâles ayant poursuivi une gestation normale.

\section{LE RÔLE DU VIEILLISSEMENT PATERNEL.}

Le rôle du mâle une fois établi, il s'agit de savoir si le vieillissement est un facteur aggravant. Une enquête déjà ancienne [9] avait conclu que le vieillissement paternel, au même titre que celui de la mère et le rang dans la naissance, pouvait être responsable de pertes foetales. Mais ce travail n'a pas été repris. On sait bien, en revanche, que les anomalies génétiques augmentent avec l'âge paternel, qu'il s'agisse d'aneuploïdies ou d'anomalies de structures des chromosomes et même de mutation dominantes ou récessives.

\section{Anomalies chromosomiques.}

Dans une population normale d'hommes âgés de 30 ans, environ $5 \%$ des spermatozoïdes présentent une non disjonction d'origne méiotique, ce qui conduit à une aneuploïdie $[10,11,12]$. Pour certains auteurs, 5 à $10 \%$ des trisomies 21 seraient ainsi d'origine masculine $[13,14]$ mais, dans ce cas, le rôle de l'âge paternel est très discuté: perceptible pour les uns $[15,16$, 17], il est actuellement inappréciable pour les autres $[14,18,19]$. Environ 50\% des Klinefelter seraient dûs, aussi, au vieillisse- ment masculin [20]. Par contre les autres aneuploïdies comme les trisomies 13 et 18 , $47 \mathrm{XXX}$ et $47 \mathrm{XYY}$ ou la monosomie $45 \mathrm{X}$ ne seraient pas liées à l'âge paternel. En ce qui concerne les anomalies de structure des chromosomes spermatiques, on a montré qu'il existait une corrélation entre leur taux et l'augmentation de l'âge [10]. Au dessus de 44 ans par exemple, il existe $13 \%$ de gamètes porteurs d'anomalies de structures. Cependant ces anomalies paraissent ne pas avoir d'effet sur la descendance [10]. Enfin, le vieillissement paternel s'accompagne d'une élévation du taux des translocations réciproques balancées d'origine paternelle chez le foetus [21].

\section{Syndromes dominants et récessifs.}

On sait depuis longtemps que le vieillissement paternel est à l'origine de mutations autosomiques dominantes se traduisant par des malformations macroscopiques comme l'achondroplasie [22], la maladie d'Apert, le syndrome de Marfan, la fibrodysplasie ossifiante progressive, etc. [1]. Si la fréquence de chacun de ces syndromes est faible, leur nombre multiplie potentiellement les risques. Ainsi, la fréquence des anomalies dues au vieillissement paternel à partir de 40 ans atteindrait 0,3 à $0,5 \%$ des naissances ce qui, selon Friedman [23], situerait le risque au niveau de la T21 pour une femme de 35-40 ans. Tandis que Hook critique ces estimations qui, selon lui, ne reflètent que les limites supérieures du phénomène [21] Lian et al [24], étudiant environ 7500 nouveau-nés anormaux, montrent que le risque d'anomalie congénitale est de $2 \%$ lorsque le père à 20 ans, $2,4 \%$ lorsqu'il en a 40 et $2,6 \%$ lorsqu'il en a 50 . Cette augmentation de $0,6 \%$ se rapproche donc des résultats de Friedman. Les mutations autosomiques dominantes peuvent également être à l'origine d'anomalies plus discrètes que les malformations: il s'agit par exemple de la neurofibromatose de Recklinghausen, syndrome le plus fréquent (1 pour 3000 naissances) dans lequel l'âge du père paraît être impliqué [25]. 
Pour certains auteurs, il semble enfin que des mutations récessives liées à l'X peuvent aussi résulter du vieillissement paternel, comme l'hémophilie A ou la myopathie de Duchenne. Dans ces cas, la première mutation apparaitrait chez le grand-père maternel, serait transmise par ses filles et s'exprimerait chez la moitié de ses petits fils [26].

\section{Origine des mutations.}

Les mutations dont on vient de parler peuvent être dues à des facteurs exogènes (rayonnements, produits chimiques) ou à des facteurs endogènes. Ceux-ci ne sont généralement pas pris en compte et, pourtant, ils sont en rapport avec les caractères de la spermatogénèse. La probabilité d'apparition des anomalies géniques est en effet beaucoup plus grande chez le mâle que chez la femelle. On explique ce fait de la manière suivante. On sait que les phases de multiplications cellulaires sont sources de mutations géniques en particulier à cause d'erreurs de recopiage du message génétique [26]. Chez la femelle, les ovogonies se sont multipliées pendant la phase foetale et c'est à partir de ce stock, sans multiplications nouvelles que, après la puberté, seront périodiquement fournis les ovocytes. Chez le mâle, au contraire, les spermatogonies, dont les multiplications avaient eu lieu pendant la phase foetale puis s'étaient arrêtées, vont se multiplier sans cesse à partir de la puberté et jusqu'à un âge très avancé (au delà de 80 ans), même si la production diminue graduellement. Pendant la période de pleine activité sexuelle, le rythme est d'environ 23 multiplications par an. Les spermatozoïdes d'un homme de 28 ans auront ainsi derrière eux, depuis la puberté, à peu près 380 divisions cellulaires, et ceux d'un homme de 35 ans à peu près 540 . On comprend mieux, à partir de ces données, que le risque de mutation augmente avec l'âge. Il est d'ailleurs remarquable que, comme l'avaient montré Schwartz et al. [27], la morphologie des spermatozoïdes (dont on sait qu'elle est génétiquement déterminée) et leur mobilité se dégradent progressivement avec le vieillissement paternel, à partir de la trentaine.

Est-ce à dire que ces différentes anomalies pourraient comme certaines trisomies, polyploïdies ou monosomies être à l'origine d'avortements ? On peut évidemment se le demander. Toutefois, et compte tenu des précautions prises pour passer d'une espèce à l'autre, notre propre expérience, chez le rat, ne plaide pas en ce sens.

En effet, le vieillissement des mêmes mâles, entre 2,5 mois et 22 mois, ne s'accompagne d'aucune réduction du nombre de petits par portée lorsque les rats sont croisés avec des femelles jeunes et d'âge constant (Tab. I), ni d'aucune malformation [28].

\section{CONCLUSION}

Compte tenu des différents résultats en présence il semble donc que l'on peut avoir des présomptions sur le rôle de l'âge paternel dans les ASR, mais pas encore de certitudes.

Pour répondre avec précision à la question, il faudrait sans doute effectuer d'une part des études rétrospectives, avec un nombre important de couples où, pour des pères jeunes et des pères âgés, le facteur féminin serait constant, ce qui n'est pas la moindre difficulté; d'autre part des études prospectives, avec exploration des spermatozoïdes et de leur carytotype dans les cas d'ASR "tout-venant" et dans ceux impliquant des hommes âgés, à la recherche d'anomalies communes, etc.

Enfin, si l'on se réfère à la courbe de la qualité des spermatozoides et à celle de la qualité du conceptus qui, entre la puberté et la maturité présentent chacune, chez l'homme et l'animal, une partie ascendante [29], on peut se demander s'il ne serait pas utile, aussi, d'étudier l'influence du très jeune âge paternel sur les ASR. 
Tableau 1. Age du père et nombre de petits par portée. 15 mâles ont été croisés, à des âges différents, avec des femelles toujours âgées de 2 mois 1/2 (2 femelles par mâle). Il n'existe aucune différence entre les nombres moyens de petits par portée correspondant aux différents âges patemels.

AGE DU PERE ET NOMBRE DE PETITS PAR PORTEE (RAT)

\begin{tabular}{lcccccc}
\hline AGE DES PERES (mois) & 2,5 & 6 & 10 & 14 & 18 & 22 \\
Nb. PERES/AGE & 15 & - & - & - & - & - \\
Nb. de MERES (2,5 mois) & 24 & 20 & 22 & 20 & 23 & 21 \\
Nb. moy. PETITS/PORTEE & $\begin{array}{c}9,1 \\
\pm 0.14\end{array}$ & $\begin{array}{c}9,2 \\
\pm 0,14\end{array}$ & $\begin{array}{c}8,7 \\
\pm 0,11\end{array}$ & $\begin{array}{c}9,6 \\
\pm 0,14\end{array}$ & $\begin{array}{c}10,3 \\
\pm 0,10\end{array}$ & $\begin{array}{l}9,7 \\
\end{array}$ \\
\hline
\end{tabular}

\section{REFERENCES}

1. EDWARDS RG. Causes of early embryonic loss in human pregnancy. Hum. Reprod., 1986, 1: 185-198.

2. GLASS RH., GOLBUS MS. Habitual abortion. Fertil. Steril. 1978, 29, 257-265.

3. MARTIN du PAN RC., DAHOUN S. Rôle du facteur masculin dans les avortements à répétition. J. Gynecol. obstet. Biol. Reprod., 1992, 21, 739-742.

4. ROSENBUSCH B., STERZIK K. Sperm chromosomes and habitual abortion. Fertil. Steril., 1991, 56, 370-372.

5. IBRAHIM M.E., MOUSSA M., PEDERSEN H. Sperm chromatin heterogeneity as an infertility factor. Arch. Androl., 1988, 21, 129-133.

6. NAROD S.A., DOUGLAS G.R., NESTMANN E.R., BLAKEY D.H. Human mutagens: evidence from paternal exposure ? Environ. Mol. Mutag., 1988, 11, 401415.

7. SAVITZ D.A., SONNENFELD N.L., OLSHAN A.F. Review of epidemiologic sudies of paternal occupational exposure and spontaneous abortion. Am. J. Ind. Med., 1994, 25, 361-383.

8. VELEZ DE LA CALLE J.C., JEGOU B. Protection by steroids contraceptives against procarbanize induced sterility and genotoxicity in male rats. Cancer Res., 1990, 50, 1308-1315.

9. SELVINS S., GARFINKE G. Paternal age, maternal age and birth order and risk of a fetal loss. Human Biology, 1976, 48, 223-230.

10. MARTIN R.H., RADEMAKER A.W. - The effect of age on the frequency of sperm chromosomal abnormalities in normal men. Am. J. Hum. Genet., 1987, 41, 484-492.

11. MARTIN R.H., BALKAN W., BURNS K., RADEMAKER A.W., LIN C.C., RUDD N.L. The chromosome constitution of 1000 human sperm: variability among normal individuals. Hum. Genet., 1985, 70, 18-24.

12. BRANDRIFF B., GORDON L., ASHWORTH L., WATCHMAKER G., MOORE II D., WYROBEK A.J., CARRANO A.V. - Chromosomes of human sperm: variability among normal individuals. Hum. Genet., 1985, 70, 18-24.

13. SHERMAN S.L., TAKAESU N., FREEMAN S., PHILIPS C., BLACKSTON R.D., KEATS B.J., JACOBS P.A., COCKWELL A.E., KURNITE D., UCHIDA I., HASSOLD T.J. - Trisomy 21: association between reduced recombination and non dysjunction. Am. J. Hum. Genet., 1990, 47, A97.

14. STYLIANOS E., ANTONARAKIS M.D. - The down syndrome collaborative group. Parental origin of the extra chromosome in trisomy 21 
as indicated by analysis of DNA polymorphisms. N. Engl. J. Med., 1991, 324, 872-876.

15. STENE J., FISHCER G., STENE E., MIKKELSEN M., PETERSEN E. - Paternal age effect in Down's syndrome. Ann. Hum. Genet., 1977, 40, 299-306.

16. MATSUNAGA E., TONOMURA E., OISHI A., KIKUCHI Y. - Reexamination of paternal age effect in Down's syndrome. Hum. Genet., 1978, 40, 259-268.

17. CECOS, THEPOT F., WACK T., SELVA J., CZYGLIK F., MAYAUX M.J. Age paternel et issues de grossesses. Expérience des CESCOS. Contracept. Fertil. Sex., 1993, 21, 388390.

18. ROTH M.P., STOLL C., TAILLEMITTE J.L., GIRARD S., BOUE A. - Paternal age and Down's syndrome diagnosed prenatally: no association in French data. Prenat. Diagn., 1983, 3, 327-335.

19. HOOK E.B., REGAL R.R. - A search for a paternal age effect upon cases of $47,+21$ in which the extra chromosome is of paternal origine. Am. J. Hum. Genet.,1984, 36, 413421.

20. HASSOLD T.J., - The origin of non-dysjunction in humans. Meeting of the International Human Genetics Society. Washington. October 1991. In: Ségolène Aymé: Epidémiologie de la trisomie 21. Les données récentes. 10e Séminaire de diagnostic anténatal des malformations. Paris (Necker), 1991.

21. HOOK E.B., SCHREINEMACHERS D.M., WILLEY A.M., CROSS P.K. Inherited structural cytogenetic abnorrnalities detected incidentally in fetuses diagnosed prenatally: frequency, paternal age associations, sex-ratio trends, and comparisons with rates of mutants. Am. J. Hum. Genet.,1984, 36, 422-443.

22. PENROSE L.S. - Paternal age and mutation. Lancet, 1955, ii, 312-313.

23. FRIEDMAN J.M. - Genetic desease in the offspring of older fathers. Obstet. Gynecol, 1981, 57, 745-749.

24. IIAN ZH., ZACK M., ERICKSON JD. Paternal age and the occurence of birth defects. Am. J. Hum. Genet., 1986, 39, 648660 .
25. KAPLAN J., TOUTAIN A. - La maladie de Recklinghausen. In 3e Sem. Genet. Clin.: affections dominantes à expression variable et Conseil Génétique, Paris, Necker, 1987, 42-52.

26. VOGEL F., RA HENBERG R. - Spontaneous mutation in man. Adv. Hum. Genet., 1975, 5, 223-318.

27. SCHWARTZ D., MAYAUX MJ., SPIRA A., MOSCATO ML., JOUANNET P., CZYGLIK F., DAVID G. - Study of a group of 484 fertile men. Part II: relation between age (20-59) and semen characteristics. Int. J. Androl., 1981, 4, 450-456.

28. AUROUX M. - Decrease of learning capacity in offspring with increasing patemal age in the rat. Teratology, 1983, 27, 141-148.

29. AUROUX M. Paternal age and development. In: Fertility and Sterility, a current overview. B. HEDON, J. BRINGER and P. MARES eds., Proceedings of the 15th World Congress on Fertility and Sterility, Montpellier, France, 17-22 Sept 1995, 321328.

\section{ABSTRACT}

\section{Paternal age and habitual abortion}

\section{Auroux.}

Paternal factors, particularly genetic ones, can play a role in habitual abortion (HA): trisomies, polyploidies and $X$ monosomy are the most frequent forms of involved abnormalities. The male professionnal occupation is able to lead to HA through genetic defects. Paternal ageing, which increases the risk of autosomic dominant syndroms, could also increase the HA risk through more and more errors in spermatogonia DNA replication. However, this possibility is not clearly established. From a general point of view, the role of a very young paternal age should be also considered.

Key words : paternal age, habitual abortion, caryotypical abnormalities, mutations. 